\title{
RELACION ENTRE EL NIVEL DE CONOCIMIENTO Y ACTITUD SOBRE VIH-SIDA DE LOS ESTUDIANTES DE LA FACULTAD DE ENFERMERIA - UNICA 2013.
}

\author{
RELATIONSHIP BETWEEN THE LEVEL OF KNOWLEDGE AND ATTITUDES ABOUT HIV AIDS \\ STUDENTS OF THE FACULTY OF NURSING - UNICA 2013.
}

Rafael Octavio Carhuayo Ascencio ${ }^{1, a}$ María Elena Figueroa Cabezudo ${ }^{2, a, b}$

\footnotetext{
${ }^{1}$ Facultad de enfermería Universidad Nacional San Luis Gonzaga de Ica.

${ }^{2}$ Hospital Augusto Hernández Mendoza

a Licenciado en enfermería, ${ }^{b}$ Especialista en Pediatría
}

\section{RESUMEN}

El VIH/SIDA sigue siendo uno de los más graves problemas de salud pública del mundo, especialmente en los países de ingresos bajos o medios. En el contagio del $\mathrm{VIH}$, el comportamiento de las personas tienen un papel determinante y la prevención consiste precisamente en el abandono de los comportamientos de riesgo junto con la adopción y mantenimiento de comportamientos preventivos. EI VIH/SIDA persiste como una enfermedad socialmente temida y causa de discriminación, evidenciándose déficit de conocimientos sobre formas de contagio, conduciendo a actitudes perjudiciales hacia quienes padecen este mal. Objetivo: Determinar la relación entre el nivel de conocimiento y la actitud sobre el VIH-SIDA de los estudiantes de Enfermería de la UNICA. Material y métodos: La población de estudio estuvo constituida por 530 estudiantes matriculados en la Facultad de enfermería en los diferentes ciclos, de los cuales se realizó un muestreo probabilístico aleatorio estratificado obteniéndose 271. La técnica de recolección de datos fue la encuesta teniendo como instrumento un cuestionario. Resultados: El nivel de conocimiento sobre VIH-SIDA fue alto alcanzando $99 \%$ (267) en tanto que la actitud fue favorable alcanzando $85 \%(230)$. Se demostró que las variables están relacionadas a través del estadístico chi $^{2}(5,99)$ rechazándose la hipótesis nula. Conclusiones: Existe relación entre el nivel de conocimientos y la actitud sobre VIH-SIDA de los estudiantes de enfermería de la UNICA 2013. El nivel de conocimientos sobre el VIH-SIDA es alto. La actitud frente al VIH-SIDA fue favorable.

Palabras clave: Virus inmuno deficiencia humana, SIDA, conocimiento, actitud.

\section{SUMMARY}

HIV/AIDS remains one of the most serious public health problems of the world, especially in countries of low and middle income. In HIV infection, the behavior of the people have a role and prevention is precisely the abandonment of risky behavior and the adoption and maintenance of preventive behaviors. HIV / AIDS remains a socially dreaded disease and causes of discrimination , showing lack of knowledge about modes of transmission, leading to negative attitudes towards those with this disease. The aim of the study was to determine the relationship between the level of knowledge and attitudes about HIV - AIDS and nursing students UNICA. Methods: The study population consisted of 530 students enrolled in the School of Nursing in different cycles, of which a random stratified probability sampling was performed yielding 271 . The technique of data collection was the survey with a questionnaire as an instrument .Results: The level of knowledge about HIV AIDS was high, reaching 99\% (267) while the attitude was favorable reaching 58\% (156). It showed that the variables are related through statistical chi2 (5.99) rejecting the null hypothesis. Conclusions: There is a relationship between the level of knowledge and attitudes about HIV-AIDS nursing students of UNICA 2013 The level of knowledge about HIV-AIDS is high.. The attitude towards HIV-AIDS was favorable.

Keywords: Virus human immune deficiency, AIDS knowledge, attitude. 


\section{INTRODUCCIÓN}

El síndrome de inmunodeficiencia adquirida (SIDA) es una enfermedad del sistema inmunológico humano causada por el virus de la inmunodeficiencia humana $(\mathrm{VIH})$. Es la enfermedad que constituye la principal causa de defunción en todo el mundo. En el pasado el VIH/SIDA era considerada una enfermedad mortal, se propaga fundamentalmente a través del contacto sexual sin protección, el uso compartido de jeringas, así como a través de transfusiones de sangre contaminadas. La investigación médica ha ayudado a comprender mejor el VIH y mejorar los tratamientos disponibles, ahora puede ser tratado con medicina, sin embargo las enfermedades infecciosas crónicas siguen siendo un problema grave (1).

Se calcula que en 2012 se infectaron unos 2,3 millones de personas, cobrando vidas de unos 36 millones de personas. Desde 2001, la incidencia anual del VIH ha disminuido en 33 países, 22 de ellos del África subsahariana. Sin embargo, está aumentando de nuevo en Europa Oriental y Asia Central, después de haber disminuido a principios de los años 2000, y también está en aumento en el Oriente Medio y el Norte de África (2). Según el informe de ONUSIDA 2011 el número de personas que mueren por causas relacionadas con el SIDA disminuyó a 1,8 millones en el 2010, desde el nivel máximo de 2,2 millones alcanzado a mediados de los años 2000.Desde 1995, se ha evitado un total de 2,5 millones de muertes en países de ingresos bajos y medianos debido al tratamiento antirretrovírico que se introdujo, según los nuevos cálculos de ONUSIDA. Gran parte de ese éxito proviene de los últimos dos años, cuando se produjo una rápida ampliación del acceso al tratamiento; solo en 2010, se evitaron 700.000 muertes relacionadas con el sida. La proporción de mujeres que viven con el $\mathrm{VIH}$ se ha mantenido estable al 50\%en todo el mundo, aunque este grupo de población es más afectado en África subsahariana $(59 \%$ de todas las personas que viven con el $\mathrm{VIH}$ ) y el Caribe (53\%).El número de personas que se infectan por el VIH sigue en descenso, en algunos países más rápido que en otros. La incidencia del VIH ha caído en 33 países, 22 de los cuales pertenecen a África subsahariana, la región más afectada por la epidemia de sida (3).

En América Latina, el total estimado de nuevas infecciones por el VIH fue de 170000 $\mathrm{y}$, en consecuencia, el número de personas que viven con el $\mathrm{VIH}$ asciende a 2 millones. Según las estimaciones, aproximadamente 77 000 personas fallecieron a causa de enfermedades relacionadas con el sida durante el último año, el número de infecciones por el VIH entre hombres es significativamente más elevado que entre mujeres, debido en gran parte a la predominancia de la transmisión sexual entre hombres (4).

El Ministerio de Salud -MINSA reporta en sus estadísticas correspondientes al 2010, que existen 23,446 casos de SIDA y 36.138 de VIH en el Perú. Sin embargo, según el modelo de estimaciones desarrollado por OMS y ONUSIDA, existen aproximadamente 76,000 personas viviendo con $\mathrm{VIH} /$ Sida en el Perú. La mayoría de los casos proceden de las ciudades y departamentos con mayor concentración urbana de la costa y selva peruanas. Lima y Callao concentran el $73 \%$ de los casos registrados. De todos los casos notificados de SIDA desde el año 1983 a la fecha, el $80 \%$ pertenece al sexo masculino y el $20 \%$ al sexo femenino. La mayoría de casos de SIDA se concentra entre jóvenes y adultos jóvenes en edad productiva (25 y 35 años). Se ha estimado que entre 11,40020,200 jóvenes (15-34 años) viven con VIH, pero solamente el $55 \%$ por ciento de ellos cuentan con el acceso al conocimiento, información y educación sobre el VIH y sobre cómo protegerse del riesgo de contraerlo (4). 
En ese marco, después de Lima y Callao, Ica es el departamento con mayor número de casos de VIH-sida a nivel nacional. De acuerdo con los datos alcanzados por la Oficina de Epidemiología de la Dirección Regional de Salud (DIRESA) de Ica, se registran desde 1987 hasta junio de 2011, 931 casos de infección de VIH y 823 casos de SIDA (5).

La actitud negativa y el estigma asociado al VIH y al SIDA es reconocido como uno de los principales obstáculos para abordar cuestiones de prevención y atención y garantizar el acceso a los servicios esenciales de salud de las personas que viven con VIH. A un nivel superficial, la escasa comprensión, los mitos sobre la transmisión, la asociación inicial de la infección con grupos socialmente marginados (como las trabajadoras sexuales y los varones homosexuales activos) y el temor al contagio y a la muerte, se encuentran entre los numerosos elementos asociados al estigma por $\mathrm{VIH} / \mathrm{SIDA}(6)$.

Los jóvenes son especialmente vulnerables a la infección por el $\mathrm{VIH}$, porque muestran menor interés que los adultos en comportarse de forma saludable, lo que ha llevado a considerarlos un grupo de alto riesgo en relación con la salud, influidos por sus creencias y valores en relación con el entorno social en el que desarrollan su vida. La percepción de lo que piensan los compañeros tiene por lo común mayor influencia en el comportamiento sexual o en cualquier otro tipo de comportamiento arriesgado, comparado con las opiniones de los padres y otros adultos. La mayoría tienen información insuficiente o algunos han escuchado hablar o reciben información errada, esto se da porque no existe muchas veces una información adecuada salud - educación por los profesores y los profesionales de salud, por lo cual surge el interés de conocer qué nivel de conocimiento tienen los estudiantes de enfermería y cuál es la actitud sobre el VIH-SIDA.

El objetivo fue determinar la relación entre el nivel de conocimiento y la actitud sobre el
VIH-SIDA de los estudiantes de Enfermería de la UNICA.

\section{MATERIAL Y MÉTODOS:}

El estudio corresponde al tipo cuantitativo, según su enfoque, descriptivo y correlacional según su alcance $y$ de diseño no experimental de corte transversal. La técnica de recolección de datos fue la encuesta y como instrumento un cuestionario el cual fue previamente validado a través de juicio de expertos y $\mathrm{R}$ de Pearson así como también fue demostrada su confiabilidad a través del Alfa de Cronbach obteniendo una confiabilidad de 0,81 y 0,65 para cada variable respectivamente. La población estuvo constituida por 530 estudiantes matriculados en la facultad de enfermería de I a $X$ ciclo, de la cual se extrajo una muestra probabilística estratificada obteniendo como resultado 271 estudiantes, en la recolección de datos se tomó en cuenta los aspectos éticos de autonomía y anonimia. Para el procesamiento de datos se tomó en cuenta el programa SPSS versión 17 en el vaciado electrónico y las pruebas estadísticas necesarias para determinar la relación de las variables, la cual fue $\mathrm{chi}^{2}$, para luego ser exportados en Microsoft Excel y elaborar los gráficos y tablas, tomando en cuenta una sumatoria simple para la primera variable, para luego establecer los intervalos en cada nivel estipulados en cada gráfico. En tanto que en la segunda variable se tomó en cuenta una sumatoria para luego establecer el promedio y hallar los valores finales establecidos como favorables $y$ desfavorables.

\section{RESULTADOS:}

El nivel de conocimiento sobre VIH-SIDA fue alto alcanzando 99\% (267 estudiantes) en tanto que la actitud fue favorable alcanzando 85\% (230 estudiantes). Se demostró que las variables están relacionadas a través del estadístico chi $^{2} \quad \mathbf{( 5 , 9 9 )}$ rechazándose la hipótesis nula. 
TABLA 1. DATOS GENERALES DE LOS ESTUDIANTES DE LA FACULTAD DE ENFERMERÍA UNICA 2013

\begin{tabular}{lrr}
\hline ACTIVIDAD SEXUAL & $\mathbf{f}$ & $\%$ \\
\hline SI & 78 & $29 \%$ \\
NO & 193 & $71 \%$ \\
TOTAL & 271 & $100 \%$ \\
USO DE CONDÓN & $\mathbf{f}$ & $\%$ \\
SI & 75 & $28 \%$ \\
NO & 196 & $72 \%$ \\
TOTAL & 271 & $100 \%$ \\
MAS DE UNA PAREJA SEXUAL & $\mathbf{f}$ & $\%$ \\
SI & 8 & $3 \%$ \\
NO & 263 & $97 \%$ \\
TOTAL & 271 & $100 \%$ \\
\hline
\end{tabular}

TABLA 2. NIVEL DE CONOCIMIENTO SOBRE VIH-SIDA SEGÚN SUS DIMENSIONES

\begin{tabular}{lrr}
\hline APECTOS GENERALES & $\mathbf{f}$ & $\%$ \\
\hline ALTO (11 a 12) & 265 & $98 \%$ \\
MEDIO (8 A 10) & 6 & $2 \%$ \\
BAJO (6 a 7) & 0 & $0 \%$ \\
\hline VIAS DE TRANSMISIÓN & $\mathbf{f}$ & $\%$ \\
\hline ALTO (11 a 12) & 88 & $32 \%$ \\
MEDIO (8 A 10) & 183 & $68 \%$ \\
BAJO (6 a 7) & 0 & $0 \%$ \\
\hline FACTORES DE RIESGO & $\mathbf{f}$ & $\%$ \\
\hline ALTO (7 a 8) & 220 & $81 \%$ \\
MEDIO (6) & 51 & $19 \%$ \\
BAJO (4 a 5) & 0 & $0 \%$ \\
\hline DIAGNÓSTICO Y TRATAMIENTO & $\mathbf{f}$ & $\%$ \\
\hline ALTO (9 a 10) & 138 & $51 \%$ \\
MEDIO (7 a 8) & 133 & $49 \%$ \\
BAJO (5 a 6) & 0 & $0 \%$ \\
\hline PREVENCIÓN & $\mathbf{f}$ & $\%$ \\
\hline ALTO (7 a 8) & 205 & $76 \%$ \\
MEDIO (6) & 66 & $24 \%$ \\
BAJO (4 a 5) & 0 & $0 \%$ \\
TOTAL & 271 & $100 \%$ \\
\hline
\end{tabular}

TABLA 3. NIVEL DE CONOCIMIENTO SOBRE VIH-SIDA EN LOS ESTUDIANTES DE LA FACULTAD DE ENFERMERÍA UNICA 2013

\begin{tabular}{lrr}
\hline NIVEL DE CONOCIMIENTO & \multicolumn{1}{c}{ f } & \multicolumn{1}{c}{$\%$} \\
\hline ALTO (42 a 50) & 267 & $99 \%$ \\
MEDIO (34 a 42) & 4 & $1 \%$ \\
BAJO (25 a 33) & 0 & $0 \%$ \\
\hline
\end{tabular}


TABLA 4. ACTITUD SOBRE VIH-SIDA SEGÚN SUS DIMENSIONES

\begin{tabular}{lcc}
\hline DIMENSION COGNITIVA & $\mathbf{f}$ & $\%$ \\
\hline FAVORABLE & 161 & $59 \%$ \\
DESFAVORABLE & 110 & $41 \%$ \\
TOTAL & 271 & $100 \%$ \\
\hline DIMENSION AFECTIVA & $\mathbf{f}$ & $\%$ \\
\hline FAVORABLE & 253 & $93 \%$ \\
DESFAVORABLE & 18 & $7 \%$ \\
TOTAL & 271 & $100 \%$ \\
\hline DIMENSIÓN CONDUCTUAL & $\mathbf{f}$ & $\%$ \\
\hline FAVORABLE & 188 & $69 \%$ \\
DESFAVORABLE & 83 & $31 \%$ \\
TOTAL & 271 & $100 \%$ \\
\hline
\end{tabular}

\section{TABLA 5. PROMEDIO GLOBAL DE LA ACTITUD SOBRE VIH-SIDA SEGÚN SUS DIMENSIONES}

\begin{tabular}{lrr}
\hline ACTITUD & \multicolumn{1}{c}{$\mathbf{f}$} & $\%$ \\
\hline FAVORABLE & 230 & $85 \%$ \\
DESFAVORABLE & 41 & $15 \%$ \\
TOTAL & 271 & $100 \%$ \\
\hline
\end{tabular}

\section{DISCUSIÓN.}

De acuerdo a los resultados obtenidos en el estudio se confronta con estudios anteriores lo cual permite afirmar lo siguiente:

Los estudiantes de enfermería en su mayoría corresponden a IX ciclo representado por un $13 \%$; tienen edades que corresponden al grupo etáreo comprendido en las edades de 20 y 21 años; el sexo femenino alcanza el 93\%; han manifestado además que aún no tienen actividad sexual en un $71 \%$ y solo el $29 \%$ manifestó haber tenido actividad física, de los cuales el $28 \%$ ha usado condón en sus relaciones sexuales y sólo un 3\% manifestó tener más de una pareja sexual. El estudio tiene aspectos similares como la edad con el antecedente de Vásquez PM. Y col (10) quienes encontraron que de la muestra, el $83.6 \%$ presentan edades de 17 a 20 años, $52.4 \%$ son del sexo masculino. La predominancia del sexo en la carrera de enfermería es mayor en todo nivel y espacio, pues para la mujer la vocación de servicio perdura desde sus inicios con Florencia Nightingale. A pesar de observar y destacar conductas sexuales saludables en la mayoría, es necesario que se fortalezcan las pautas relacionadas a la toma de conciencia en torno al cuidado de la salud, tanto de la persona como de quienes nos rodean, desde el entorno familiar y universitario dado que los jóvenes, etapa de vida a la cual pertenece la mayoría aún se encuentra en proceso de 
maduración, susceptible a cambios de acuerdo al entorno.

Así mismo, en relación a la primera variable nivel de conocimiento sobre los aspectos generales del VIH-SIDA como concepto, fisiopatología y síntomas fue alto alcanzando un $98 \%$ y solo el $2 \%$ fue medio, sobre las vías de transmisión del VIH-SIDA fue medio alcanzando un $68 \%$, sobre los factores de riesgo del $\mathrm{VIH}$-SIDA fue alto alcanzando un $81 \%$; sobre el diagnóstico y tratamiento del VIH-SIDA fue alto alcanzando un 51\% siendo el nivel de conocimiento sobre prevención del VIH-SIDA fue alto alcanzando un $76 \%$.

Muchos estudios se han realizado tanto a adolescentes y jóvenes respecto al VIH/SIDA puesto que como se sabe constituyen una población en riesgo, sin embargo en los futuros profesionales de enfermería el conocimiento es básico no solo para el cuidado propio sino para la educación que debe impartir a la población como parte de su profesión. Así tenemos que Barrueta Blanco R. (7). en su estudio realizado en un grupo de estudiantes escolares encontró que el nivel de conocimiento fue medio, resaltando que en cuanto al concepto de VIH/SIDA el nivel es alto. A diferencia del estudio de Ruiz $F$ (8). Respecto al nivel de conocimiento acerca de $\mathrm{VIH} /$ sida, de los 60 estudiantes encuestados existen un $86 \%$ que tiene alto nivel de conocimiento. Lo cual difiere totalmente con el estudio realizado por Cajo y col (9) obtuvo en una muestra de estudiantes escolares que el nivel de conocimiento sobre VIH/SIDA fue bajo tanto particulares como estatales. En todos los niveles desde el escolar es necesario fortalecer el aspecto educativo sanitario en torno al VIH/SIDA, temas comprendidos como parte de la educación sexual. Finalmente el nivel de conocimiento de manera global sobre VIH-SIDA fue alto alcanzando un $99 \%$ y solo el $1 \%$ fue medio en los estudiantes de enfermería, resultados diferentes al estudio de Vásquez PM. Y col (10) en su estudio encontraron que existe una insuficiente información sobre sexualidad y sobretodo en la prevención de las ITS, muestran a nuestra población como susceptible a padecer enfermedades relacionadas con el sexo y las consecuencias de las mismas, concluyendo que el nivel de conocimiento es adecuado sin embargo el comportamiento sexual es de riesgo. Estos resultados son buenos en el sentido cognitivo, sin embargo alarmantes en torno a la práctica lo cual está ligada sin duda alguna la actitud que tienen los jóvenes, el saber demasiado no implica necesariamente que su conducta sea favorable. Respecto a la segunda variable, actitud frente al VIH/SIDA, se obtuvo que según su dimensión cognitiva fue favorable alcanzando un 59\%; según su dimensión cognitiva fue favorable alcanzando un 93\%; según su dimensión conductual fue favorable alcanzando un $69 \%$, lo cual de manera global refleja un $85 \%$ como favorable, esta actitud se ve reflejada no solo en el cuidado de la persona sino en este grupo de estudio implica el comportamiento frente a personas que pueden tener VIH-SIDA y que al interrelacionarse con ellos reflejen una actitud favorable, sin estigmas ni llegar a la discriminación. Resultados diferentes encontró Barrueta Blanco R. (7) en su estudio realizado en un grupo de estudiantes escolares encontró que el nivel de conocimiento fue medio sin embargo las prácticas sexuales son riesgosas lo cual evidencia una actitud desfavorable en torno a la enfermedad. Asimismo, Fuster MJ y col (11) del análisis descriptivo muestran que en España son una realidad el prejuicio y las actitudes discriminatorias hacia las personas con VIH. Se ha hallado que las creencias erróneas sobre las vías de transmisión, la culpabilización de las personas con $\mathrm{VIH}$, la percepción de gravedad de la infección y la lejanía percibida de personas con VIH son importantes variables que guardan una estrecha relación con los sentimientos y con las actitudes de la población. Este estudio revela realidades vividas por la misma autora, paciente de esta enfermedad, si analizamos la situación desde el punto de vista económico, social y cultural, España nos lleva 
mucha ventaja en dichos aspectos sin embargo aún es predominante, dichos aspectos nos llevan a considerar que las cantidades y resultados obtenidos no son permanentes, si los estudiantes de enfermería que empiezan y conocen en su mayoría los aspectos básicos del VIH-SIDA están manifestando un conocimiento satisfactorio tanto como la actitud, es necesario que se fortalezca tal aspecto a fin de valorar, mantener y/o mejorar aún más las actitudes positivas hacia el cuidado de su salud y el de las personas que la padecen. $\mathrm{Si}$ esto es necesario en un grupo de jóvenes que tienen conocimiento previo del tema, deja entrever que la población común necesita aún más del fortalecimiento de tales variables, lo cual debe tomarse en cuenta y trabajar el aspecto preventivo promocional.

Existen estudio que confirman que tomando en cuenta ambas variables se puede obtener resultados favorables, así tenemos que Fuster MJ y col (11) concluye que existe estrecha relación entre las creencias y actitudes hacia las personas con $\mathrm{VIH}$. Gutiérrez N. y col (10) demuestran que la labor de la educación en la adquisición de conocimiento conlleva a adquirir conductas responsables en la sexualidad.

Enfermería está llamada a trabajar en la prevención de esta enfermedad, además de los sufrimientos que impone a las personas y sus familias, la epidemia afecta profundamente el tejido social y económico de las sociedades. EI VIH/SIDA constituye una amenaza mayor para el mundo del trabajo: afecta al sector más productivo de la población activa, reduce los ingresos e impone costos elevados a las empresas de todos los sectores como consecuencia de la disminución de la productividad, del aumento del costo de la mano de obra y de la pérdida de trabajadores calificados y con experiencia. Por otra parte, el VIH/SIDA menoscaba los derechos fundamentales en el trabajo, sobre todo debido a la discriminación y el rechazo de que es objeto la gente que vive con el $\mathrm{VIH} / \mathrm{SIDA}$ o se ve afectada por el mismo. La epidemia y sus efectos aquejan más cruelmente a los colectivos vulnerables, incluidos los niños y las mujeres, aumentan por ende las desigualdades de trato existentes entre hombres y mujeres $y$ exacerban el problema del trabajo infantil. El trabajo aún está pendiente en la población en torno al reconocimiento de que el VIH/SIDA es un problema que afecta al lugar de trabajo, la no discriminación en el empleo, la igualdad de trato entre hombres y mujeres, el diagnóstico y la confidencialidad, la prevención, la asistencia y el apoyo como base para enfrentar la epidemia en el lugar de trabajo. En estos aspectos todos estamos llamados a contribuir y enfermería lidera dicho trabajo.

\section{CONCLUSIONES}

- Existe relación entre el nivel de conocimientos y la actitud sobre VIH-SIDA de los estudiantes de enfermería de la UNICA 2013.

- El nivel de conocimientos sobre el VIHSIDA es alto en los estudiantes de la facultad de enfermería resaltándose que en cada una de sus dimensiones: Aspectos generales, factores de riesgo, diagnóstico y tratamiento y prevención fue alto, sólo en la dimensión considerada las vías de transmisión fue medio, sin embargo a nivel global el nivel de conocimiento fue alto.

- La actitud frente al VIH-SIDA fue favorable en cada una de sus dimensiones, esta conducta abarca no solo el cuidado propio de la persona sino también en torno a paciente que padece la enfermedad, lo cual es favorable.

\section{Correspondencia:}
Lic. Rafael Carhuayo Ascencio
Correo electrónico:
rafael_carhuayo@hotmail.com 


\section{REFERENCIAS BIBLIOGRÁFICAS}

1. Salud y Familia. VIH: Generalidades del VIH. [Internet]. Perú: 2010. [Fecha de acceso10 de Marzo de 2013]. URL Disponible en: http://www.saludyfamilia. com/enfermedades/enfermedades-

sexuales/vih/

2. Organización Mundial de la Salud. Datos fundamentales sobre la epidemia mundial de VIH y los progresos realizados en 2010 . [Internet]. 2011. [Fecha de acceso12 de Marzo de 2013]. URL disponible en: http://www.who.int/hiv/pub/progress_report 2011/global_facts/es/index.html

3. ONUSIDA. Informe para el día mundial del SIDA. [Internet]. Suiza. 2011. [Fecha de acceso 12 de Marzo de 2012]. URL disponible en: http://www.unaids.org len/media/unaids/contentassets/documents lunaidspublication/2011/JC2216 WorldAID Sday report 2011 es.pdf.

4. Sistema de las Naciones Unidas en el Perú. Lucha contra el VIH/SIDA. [Internet]. Perú. 2009. [Fecha de acceso 14 de Marzo de 2012]. URL disponible en:

http://www.onu.org.pe/Publico/infocus/luch acontrasida.aspx.

5. COREMUSA - ICA. Estadística Regional del VIH/SIDA. [En línea]. Perú. 2011. [Fecha de acceso 14 de Marzo de 2012]. URL disponible en: http://coremusaica.blogspot.com/2011/10/e stadisticas-regionales-del-vihsida.html.

6. Barrueta R. Nivel de conocimiento sobre el $\mathrm{VIH} / \mathrm{SIDA}$ y las prácticas sexuales de riesgo, en los adolescentes del 5to año de la Institución Educativa Teodosio Franco García, Ica. [Tesis para optar título profesional de Licenciada de Enfermería]. Perú: Universidad Privada San Juan
Bautista. [En línea]. 2010. [Fecha de acceso 20 de Marzo de 2012].

7. Ruiz F. Nivel de conocimiento sobre VIH/SIDA y medidas de prevención en estudiantes de 5 año de secundaria de la Institución Educativa Raúl Porras Barrenechea de la provincia de Palpa Ica.[Tesis para optar título profesional de Licenciada de Enfermería]. Perú: Universidad Privada San Juan Bautista.

8. Cajo y Colaboradores "conocimiento sobre VIH-SIDA en alumnos del I ciclo de Enfermería de la UNSLG de Ica; 2007.

9. Fuster M. Creencias y actitudes de la población española hacia las personas con VIH. España; 2010.

10. Gutiérrez N. Conocimientos sobre percepción del riesgo del VIH/SIDA en adultos jóvenes de la enseñanza superior del Municipio Morón.[Tesis para optar título profesional de Licenciada de Enfermería]. Bolivia. 2007.

11. Vásquez P. et al. Conocimiento en $\mathrm{VIH} / \mathrm{SIDA}$ y comportamiento sexual en estudiantes de la UNAP, Iquitos.[Tesis para optar título profesional de Licenciada de Enfermería]. Perú: Universidad Nacional de la Amazonía Peruana. 2009.

Recibido: 13/02/14

Aprobado para Publicación: 31/05/14 